\title{
Proposal for improvement the welding process of the micro- USB connector on the mother board on tablets
}

\author{
Cristiano Mourão da Fonseca ${ }^{1}$, Jandecy Cabral Leite ${ }^{2}$ \\ Carlos Alberto de Oliveira Freitas ${ }^{3}$, Antonio da Silva Vieira ${ }^{4}$, Roberto Tetsuo Fujiyama ${ }^{5}$ \\ ${ }^{1}$ M.Sc.,Instituto de Tecnologia e Educação Galileo da Amazônia, Brasil. cristianomourao@yahoo.com.br \\ ${ }^{2}$ Dr.Departamento de Pesquisa. Instituto de Tecnologia Galileo da Amazônia, Brasil. jandecycabral@hotmail.com \\ ${ }^{3}$ M.Sc.,Instituto de Tecnologia Galileo da Amazônia , Brasil .caofreitas@yahoo.com.br \\ ${ }^{4}$ M.Sc., Instituto de Tecnologia Galileo da Amazônia, Brasil. adasilvavieira@gmail.com \\ ${ }^{5}$ Dr., Faculdade de Engenharia Mecânica. Programa de Pós-Graduação em Engenharia Industrial. Universidade Federal do Pará \\ (UFPA).fujiyama@ufpa.br
}

\begin{abstract}
The current electrical and electronic products industry have features extremely dynamic and with constant search for improvement in their manufacturing processes, seeking greater efficiency and lower costs. In the manufacturing process of the main board on tablets, in the area of welding technology, there was a problem in the micro USB connector soldering process causing high defect rates. The purpose of this article was to conduct assessment and proposal for improvement in this process. The methods and techniques used were quality tools PDCA cycle, cause and effect diagram and 5W2H. Among these solutions, the choice was to implement pallets of durestone to maintain the alignment of the connector on the reflow soldering oven. The results show a reduction in failure rates from $12.40 \%$ to $0.09 \%$ in the production line, which avoided the institution generate a total of 24,800 defective boards on the total to be produced. The total cost savings from logistics repair of these boards was approximately $70.002,80$. USD
\end{abstract}

Keywords: weldability; quality tools; SMT process; tablet.

\section{Propuesta de mejora en el proceso de soldadura del conector micro-USB en la placa madre en tablets}

\section{RESUMEN}

La industria actual de productos electro-electrónicos presenta características extremamente dinámicas y de constante búsqueda del perfeccionamiento en sus procesos de fabricación, procurando una mayor eficiencia y reducción de los costos. En el proceso de fabricación de la placa principal en tablets, en el área de tecnología de soldadura, había una problemática en el proceso de soldadura del conector micro USB generando altos índices de defectos. El objetivo de este artículo fue realizar la evaluación y propuesta de mejoras en ese proceso. Los métodos y las técnicas utilizadas fueron las herramientas de la calidad del ciclo PDCA, diagrama de causa y efectos y $5 \mathrm{~W} 2 \mathrm{H}$. Entre las soluciones presentadas, se escogió la implantación de pallets de durestone para mantener la alineación del conector en la soldadura en el horno de refusión. Los resultados obtenidos muestran una reducción de los índices de fallas en la línea de producción de un 12,40\% para un 0,09\%, lo que evitó a la institución generar un total de 24.800 placas defectuosas del total a ser producido. El costo total economizado con la logística de reparación de esas placas fue de aproximadamente $70.002,80$. USD

Palabras clave: soldabilidad; herramientas de calidad; proceso SMT; tablet.

\section{INTRODUCCIÓN}

La actual economía de mercado, caracterizada por la elevada competitividad entre las empresas, fuerza a la industria de electro-electrónicos a estar siempre en constante transformación en sus procesos de fabricación para superar a los concurrentes de mercado. Hay una constante busca de productos innovadores y perfeccionamiento de procesos productivos teniendo siempre como objetivo la busca de la excelencia en patrones de calidad aliado a un aumento de productividad y eficiencia. Las empresas están siempre en proceso de mejora continua que es una técnica de cambio organizacional lenta, suave e ininterrumpida, centrada en las actividades en grupo de las personas (equipo). Tiene por objetivo aumentar a calidad de los productos y servicios dentro de programas a largo plazo. Su foco es la mejora gradual y continua, a través de la colaboración y participación de las personas, para 
que realicen sus tareas un poco mejor cada día [1]. Es en ese contexto que las empresas utilizan herramientas y prácticas administrativas y gerenciales para la optimización de procesos y productos como el PDCA, que es una herramienta de control y mejora de procesos, el diagrama de causa y efecto, que es una técnica que expresa la relación existente entre un efecto y las posibles causas de un determinado evento, y el $5 \mathrm{~W} 2 \mathrm{H}$ el cual es utilizado para auxiliar en la planeación y acompañamiento de soluciones (plan de acción).

En el proceso de fabricación de productos electroelectrónicos uno de los principales desvíos de producción los cuales causan aumento de los costos de producción son los defectos, sean ellos funcionales o estéticos, que son definidos como la unidad de producto que no satisfacen una o más de las especificaciones para ese producto [2]. En una empresa del polo industrial de Manaos (PIM) productora de la placa principal de las tablets; que son equipos leves, con gran capacidad de procesamiento y autonomía de batería, permite al usuario la lectura de textos, navegación en internet para acceder a sitios, email, vídeos, además de la posibilidad de instalación de aplicativos y software que expanden el uso del equipo [3]; En la fase de SMT se percibió una problemática en la soldadura del conector micro USB causando niveles de defectos encima de los patrones establecidos por la institución. Las fallas hacían que las metas de producción y calidad no fueran alcanzadas y hubiera un aumento de demanda de técnicos de reparación, operadores de prueba, además del crecimiento de la cantidad de rechazos por exceso de re-trabajo.

De esa forma, fue realizada una investigación en forma de estudio de caso la cual tuvo gran importancia, pues generó nuevos conocimientos en el área de procesos de fabricación de la placa principal de las tablets que es una nueva y promisoria rama. La misma tuvo como objetivo analizar el proceso de soldadura del conector micro USB, específicamente en la problemática presentada, en busca mejoras en el proceso para generar reducción en los índices de defectos, reducción de costos, garantizar la satisfacción de los clientes y el alcance de las metas de productividad y calidad. ¿Con relación al problema presentado, cuál sería la mejor solución evaluándose el contexto del trabajo?

\section{REVISIÓN BIBLIOGRÁFICA}

\section{II.1. TECNOLOGÍA DE MONTAJE SMT}

Debido a la tenaz concurrencia entre las empresas es grande la búsqueda de nuevas tecnologías para perfeccionar los procesos y reducir los costos. La "revolución" en la electrónica ha sido sustentada por medio del desarrollo de productos con aumento de la miniaturización, mayor funcionalidad, la mejora de la confiabilidad y reducción de costos de fabricación. De hecho, las innovaciones en las metodologías de montaje de electrónicos ha mantenido bajos costos de fabricación a cada nueva generación de productos [4]. En la industria electrónica, la última gran innovación fue el advenimiento de la tecnología de montaje en superficie (Surface Mount Technology, SMT) en la cual los componentes denominados dispositivos de montaje en superficie (Surface Mounted Device, SMD) son montados directamente en la superficie de las placas a través de máquinas montadoras automáticas.

La tecnología SMT es considerada la evolución del proceso denominado a través de agujero y pin (Pin Through Hole PTH) en la cual los componentes son insertados manualmente o automáticamente en las placas en agujeros metalizados siendo soldados por máquinas de onda por el lado opuesto al cual fueron insertados. La creciente demanda de dispositivos electrónicos ha hecho del proceso de fabricación de placas de circuito impreso una promisoria industria en las últimas décadas. Como la demanda de placas aumenta, la industria se torna más dependiente de la alta velocidad de acoplamiento utilizando la tecnología de montaje SMD la cual viene substituyendo la tecnología PTH [5]. La figura 1 muestra el proceso SMT (componentes montados) en a) y PTH (componentes insertados) en b).

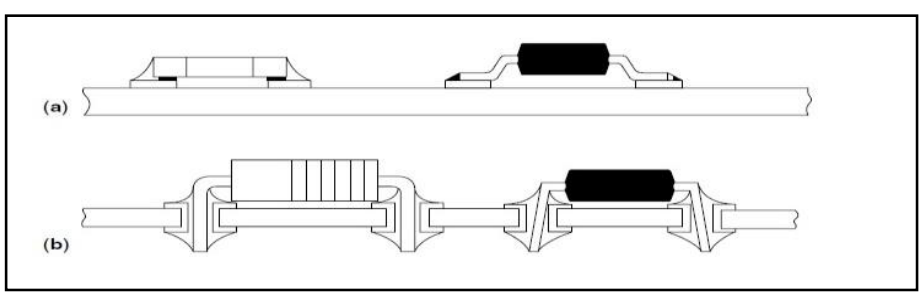

Figura 1 - Proceso a) SMT, b) PTH [6].

Entre las principales ventajas del proceso SMT en relación a su antecesor se puede destacar:

-Los componentes son menores (miniaturizados), ocupan menos espacio y son más leves posibilitando la fabricación de dispositivos portátiles como teléfonos y tablets;

-El montaje automático es más preciso y menos susceptible a errores de montaje;

-Menor costo de producción (montaje automático), y;

- Mejor resistencia mecánica.

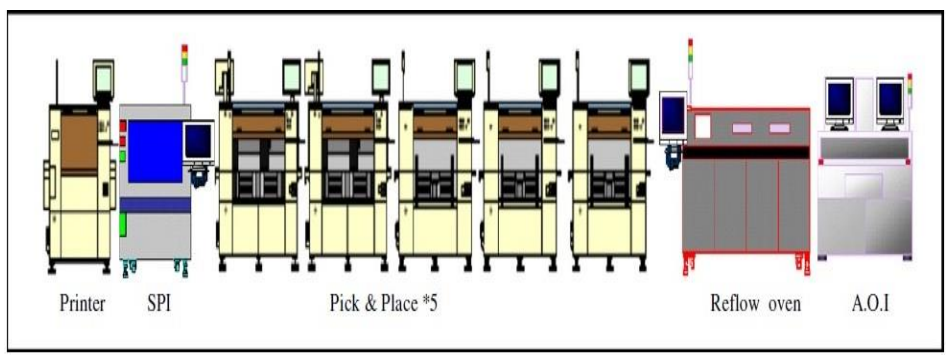

Figura 2 - Layout de una línea SMT [7].

El proceso de soldadura SMT está compuesto por equipamientos que trabajan de forma sincronizada y continua, siendo dispuestos de modo a formar líneas de producción SMT, conforme se muestra en la figura 2, el proceso está compuesto por las siguientes etapas: 
1 - Printer - Son máquinas responsables de depositar la pasta de soldadura en la superficie de la placa utilizando una matriz metálica denominada esténcil como se muestra en la figura 3.

2 - Solder Paste Inspection (SPI) - Son máquinas que hacen inspección del volumen de la pasta de soldadura depositada en la placa.

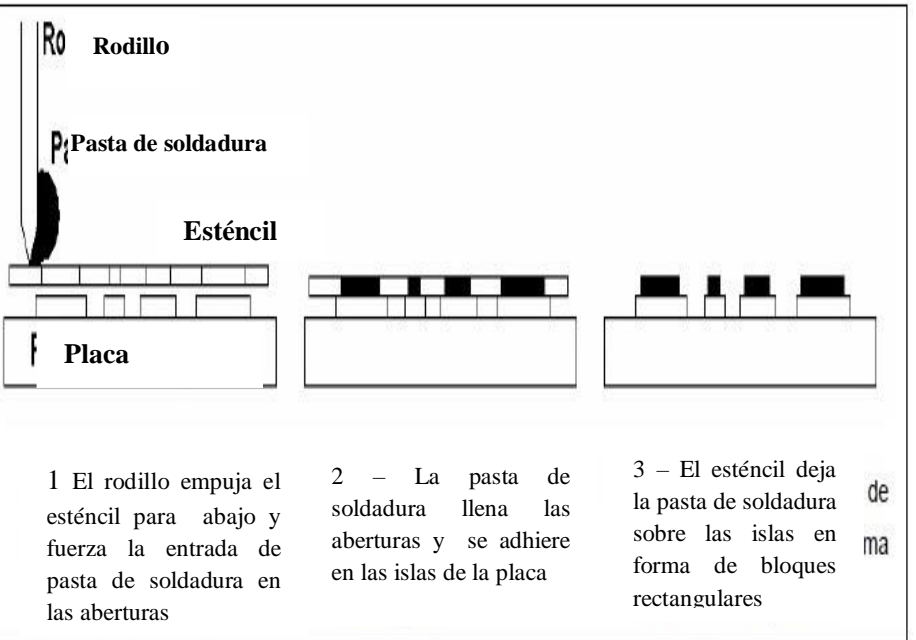

Figura 3 - Proceso de deposición de la pasta de soldadura [8].

3 - Pick \& Place (tomar e insertar) - Son máquinas de alta precisión responsables por la inserción de los componentes sobre la pasta de soldadura.

4 - Reflow Oven - La placa es colocada en un horno de refusión para que la soldadura pase del estado líquido al sólido, soldando los componentes en la superficie de la placa. El horno posee una estera la cual transporta la placa en su interior, pasando por unos "steps" de temperatura en función del tiempo denominado perfil térmico el cual está formado por cuatro fases distintas como muestra la figura 4 [9].

- Precalentamiento: la temperatura es elevada hasta el punto de evaporación de los solventes en la pasta de soldadura;

- Desgasificación: la temperatura es elevada lentamente con el objetivo de activar el flujo e igualar la temperatura en la placa;

- Refusión: la temperatura es elevada para fundir la soldadura formando entonces las uniones soldadas;

-Enfriamiento: la temperatura es reducida gradualmente hasta llegar a la temperatura ambiente.

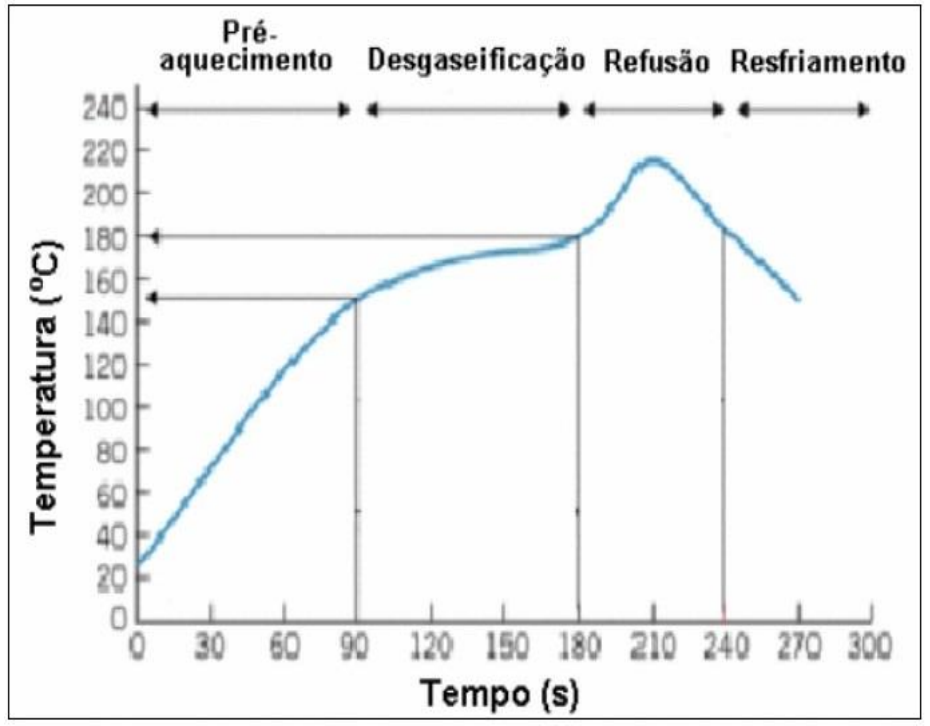

Figura 4 - Perfil térmico típico en un horno de refusión [10].

5 - Automatic Optical Inspector (A.O.I) - Son equipos que hacen la inspección óptica automática cuyo objetivo es detectar defectos de montaje. El proceso consiste en la captura inicial de la imagen en una placa de referencia y registrado en memoria. A continuación las piezas de la producción son leídas y comparadas con esa placa patrón en busca de algún defecto como ausencia de componente, componente erróneo insertado, componente con polaridad invertida y componente dislocado.

\section{II.2. LAS HERRAMIENTAS DE LA CALIDAD}

\section{II.2.1. CICLO PDCA}

PDCA es una sigla para Plan, Do, Check y Action, también es conocido como ciclo de Deming, que es un modelo de gestión de calidad clásico promovido y practicado en el Japón por Dr. W. Edward Deming, un especialista americano de gestión de la calidad [11]. En la década de los años 50 el PDCA se tornó muy popular debido a la aplicación de este método en la próspera industria japonesa realizada por Deming, el que generó una gran contribución al desarrollo de la calidad de los productos japoneses.

El ciclo PCDA es una herramienta ampliamente utilizada para análisis, resolución de problemas y toma de decisión, puede ser aplicado también para el mantenimiento manutención de metas o alcance de nuevas metas. En las empresas posee aplicaciones en diversas áreas como alcance de metas globales y estratégicas, metas dirigidas a departamentos o grupos individuales. Es considerada una metodología rápida, eficaz y de simple entendimiento, pudiendo, por tanto, ser utilizado por colaboradores de cualquier nivel de conocimiento.

El PDCA es subdividido en cuatro etapas como se describe en la figura 5 : 


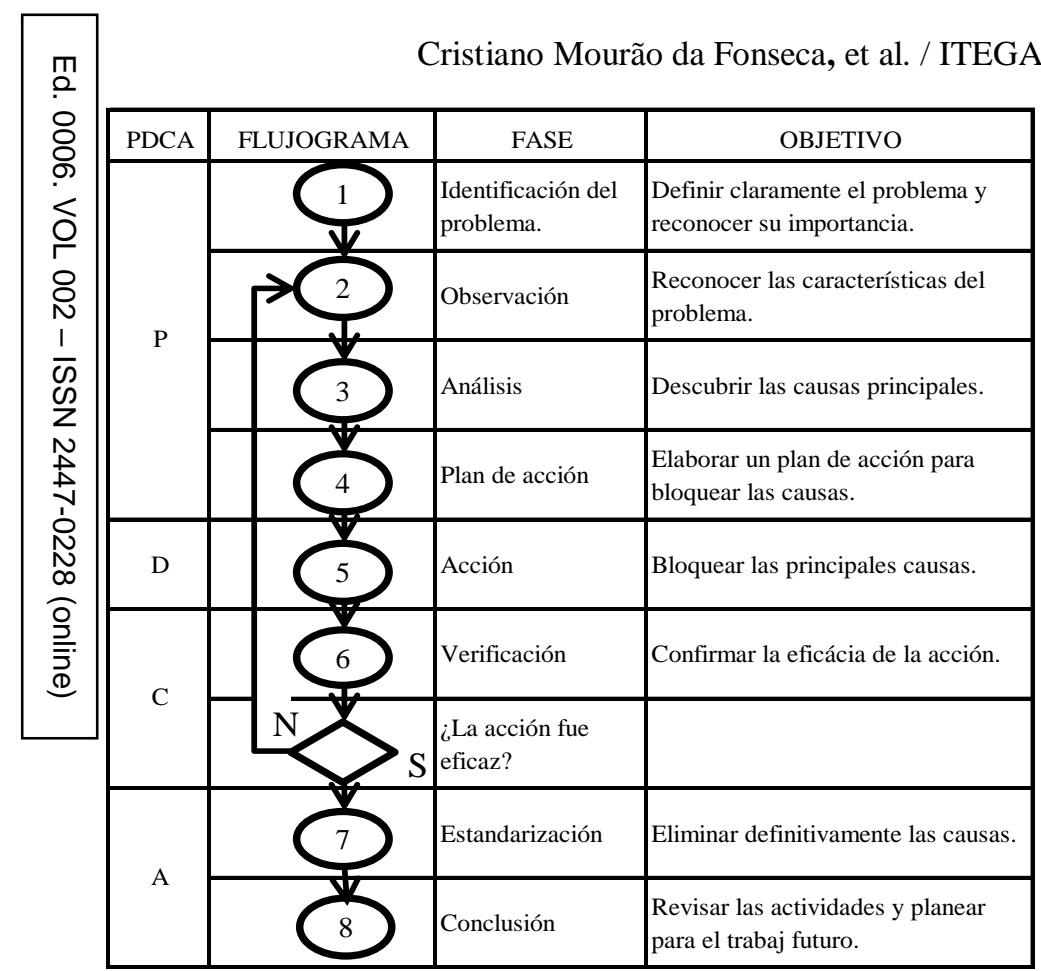

Figura 5 - Ciclo PDCA [12].

1 - Planeamiento - $(P L A N)$ - se realiza la verificación de lo que ya fue hecho, lo que debe ser alcanzado y las especificaciones y atribuciones a ser definidas [13]. Considerada la fase más importante, el planeamiento es donde el plano es trazado, el problema es identificado y detallado. Ocurre el análisis del fenómeno y del proceso identificando las causas. Posteriormente es elaborado el plan de acción para alcanzar las metas.

2 - Ejecución - $(D O)$ - Esta etapa es caracterizada por la ejecución de las acciones identificadas anteriormente. Para esto, es preciso entrenar y orientar a todos los involucrados.

3 - Verificación - $($ CHECK $)$ - En esta fase se verifica la ejecución del planeamiento de la calidad y se estudia si el resultado concuerda con la expectativa [14].

4 - Actuar correctivamente - (ACT) - A partir de los resultados obtenidos, es preciso actuar correctivamente sobre las metas no alcanzadas y establecer patrones sobre los resultados alcanzados. En caso necesario, se puede girar el ciclo PDCA nuevamente a partir del inicio, planeamiento en un proceso de mejora continua y perfeccionamiento de los procesos.

Para [15] el ciclo PDCA proporciona una busca continua por un mejor método de hacer las cosas. Al seguir este ciclo, se espera que los resultados sean obtenidos y también que el propio proceso será mejorado en una espiral ascendente. Debido a las cuatro continuas etapas del ciclo, los problemas son resueltos y el nivel de calidad aumenta durante cada ciclo. A través de los ciclos constantes del PCDA, el nivel de calidad mejora de forma continua [16] como muestra la figura 6.

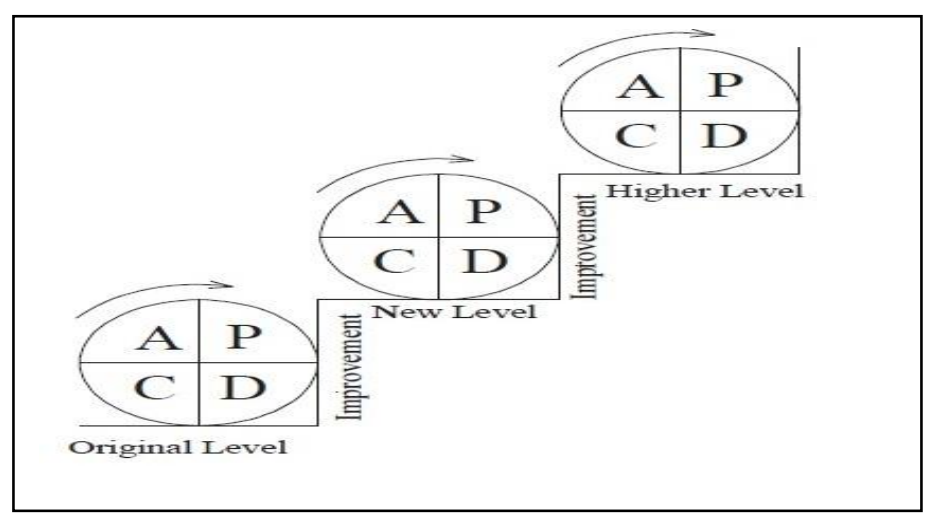

Figura 6 - Ciclo PDCA - Proceso de mejora continua [17].

\section{II.2.2. DIAGRAMA DE CAUSA Y EFECTO}

El diagrama de Ishikawa fue inventado por Kaoru Ishikawa, que fue uno de los pioneros en técnicas de gestión de la calidad en el Japón en la década de 1960. El diagrama es considerado una de las siete herramientas básicas de control de calidad. Es también conocido como diagrama de espina de pescado, debido a su forma. La "cabeza del pescado" representa el problema principal. Las posibles causas del problema, generalmente derivadas de sesiones de "brainstorming" o de investigación, son indicadas en las "espinas del pescado" del diagrama [18]. Esta herramienta expresa la relación de causa y efecto por medio de saetas, para que la causa raíz pueda ser determinada de forma sistemática [19]. El diagrama está compuesto por seis subdivisiones de posibles orígenes de los problemas denominadas "6 Ms": mano de obra, método, materia prima, máquina, material/medida y medio ambiente como muestra la figura 7 .

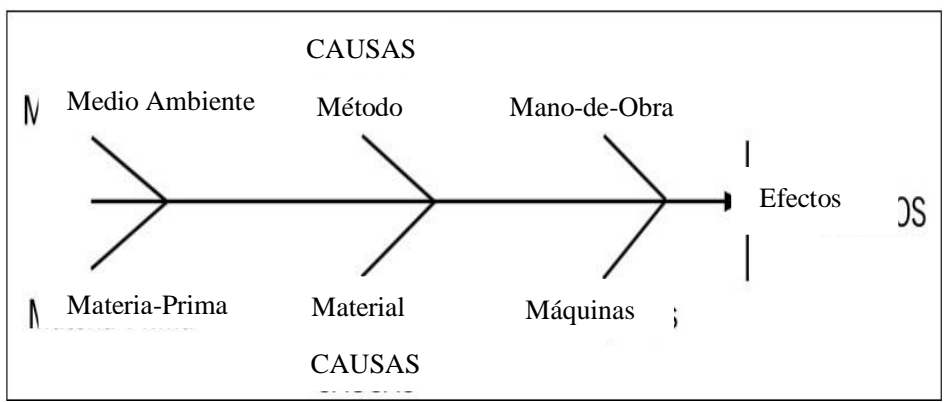

Figura 7 - Modelo gráfico del diagrama de Ishikawa [20].

\subsubsection{W2H / 5W1H}

El $5 \mathrm{~W} 1 \mathrm{H}$ sugiere que el problema puede ser analizado en base a 6 aspectos, que son por qué, qué, quién, cuándo, dónde y cómo. La esencia del $5 \mathrm{~W} 1 \mathrm{H}$ es analizar el problema sistemáticamente, incluyendo la esencia del objeto (el qué), la esencia del asunto (quién), las formas de existencia del problema en el tiempo y en el espacio (cuándo, dónde), la solución del problema (cómo) [21]. El 5W2H es una herramienta utilizada para la identificación y el mapeado de actividades a ser desarrolladas para alcanzar un determinado resultado. El nombre 


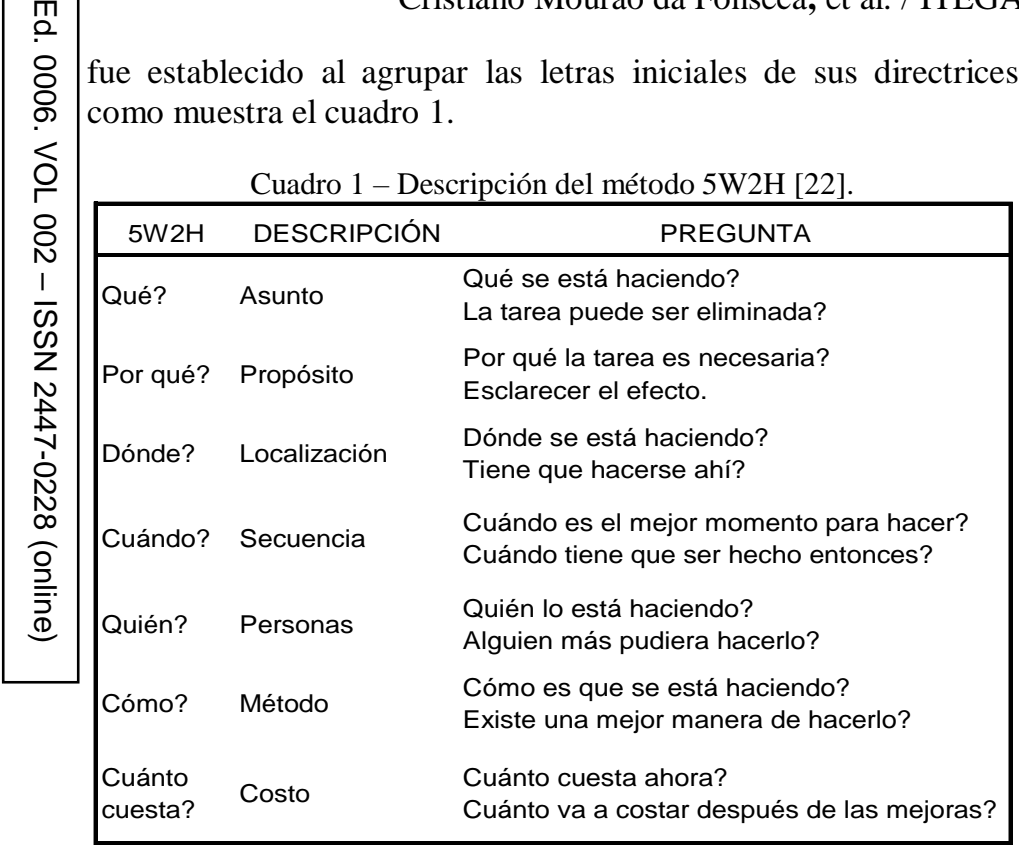

\section{MATERIALES Y MÉTODOS}

Los Materiales en el análisis de piezas defectuosas fue utilizado el microscopio óptico a fin de recolectar imágenes con mayor riqueza de detalles y de esa forma detectar las fallas en el proceso de soldadura del conector micro USB.

\section{III.1. MÉTODOS - USO DEL CICLO PDCA.}

La metodología utilizada para el desarrollo de este trabajo está basada en el ciclo PDCA que consiste en una de las herramientas más utilizadas para análisis y solución de problemas, pudiendo ser aplicada en las más diversas ramas de la industria y se caracteriza por la simplicidad de utilización. Las fases del PDCA fueron secuencialmente aplicadas en el tratamiento del problema a fin de corregirlo.

\section{III.2. PLANEAMIENTO}

En la fase del planeamiento, fue realizado el análisis en detalle del problema en la cual se obtuvo informaciones directamente en el ambiente de trabajo. Esta etapa fue subdividida en las siguientes etapas:

- Colecta de datos

La colecta de datos primeramente fue hecha a través de entrevistas con los operadores y técnicos de Ingeniería en la cual se obtuvieron los datos como se muestra en el cuadro 2.
Cuadro 2 - Resultado de la entrevista con los operadores y técnicos de Ingeniería

\begin{tabular}{|l|l|}
\hline $\begin{array}{l}1 \quad \text {-Cuál es la } \\
\text { característica } \\
\text { problema? }\end{array}$ & $\begin{array}{l}\text { El conector micro USB después } \\
\text { de pasar por el horno de refusión } \\
\text { estaba quedando desalineado. }\end{array}$ \\
\hline $\begin{array}{l}\text {-Dónde fue } \\
\text { observada la falla? }\end{array}$ & $\begin{array}{l}\text { En la etapa de pruebas } \\
\text { funcionales. }\end{array}$ \\
\hline $\begin{array}{l}3 \text { - Quién detectó el } \\
\text { defecto? }\end{array}$ & $\begin{array}{l}\text { Los operadores de las pruebas } \\
\text { funcionales y los técnicos de } \\
\text { Ingeniería. }\end{array}$ \\
\hline $\begin{array}{l}4-\text { Había registros de } \\
\text { ocurrencias } \\
\text { anteriores? }\end{array}$ & $\begin{array}{l}\text { No había registros de defectos en } \\
\text { otros productos. }\end{array}$ \\
\hline $\begin{array}{l}5-\text { La falla ocurre en } \\
\text { el 100\% del total } \\
\text { producido? }\end{array}$ & $\begin{array}{l}\text { La falla ocurría en una parte del } \\
\text { total producido. }\end{array}$ \\
\hline $\begin{array}{l}\text { 6- Cómo era corregida } \\
\text { la falla? }\end{array}$ & $\begin{array}{l}\text { Remover el conector y soldar } \\
\text { nuevamente en la posición } \\
\text { correcta. }\end{array}$ \\
\hline
\end{tabular}

Acto seguido fue realizada una investigación documental en los sistemas de registro de calidad y productividad, observándose que existían índices de defectos por encima de los patrones establecidos por la empresa que es de un el máximo de $2 \%$ y que estaban relacionados al proceso de soldabilidad del conector micro USB en la placa principal en tablets. El resultado de la investigación se muestra en la tabla 1:

Tabla 01 - Índices de fallas en la situación actual.

\begin{tabular}{|l|l|l|}
\hline $\begin{array}{l}\text { PRODUCIÓN } \\
\text { TOTAL }\end{array}$ & $\begin{array}{l}\text { CANTIDAD } \\
\text { DE DEFECTOS }\end{array}$ & $\begin{array}{l}\text { ÍNDICE } \\
\text { DE FALLAS }\end{array}$ \\
\hline 1000 PLACAS & 124 & $12,40 \%$ \\
\hline
\end{tabular}

- Análisis del problema

A partir de los dados obtenidos sobre el problema en la condición actual fue creado el diagrama de causa y efecto en el cual fue posible identificar las causas probables de la falla, y entre ellas, la causa raíz del problema. El diagrama se muestra en la figura 8 .

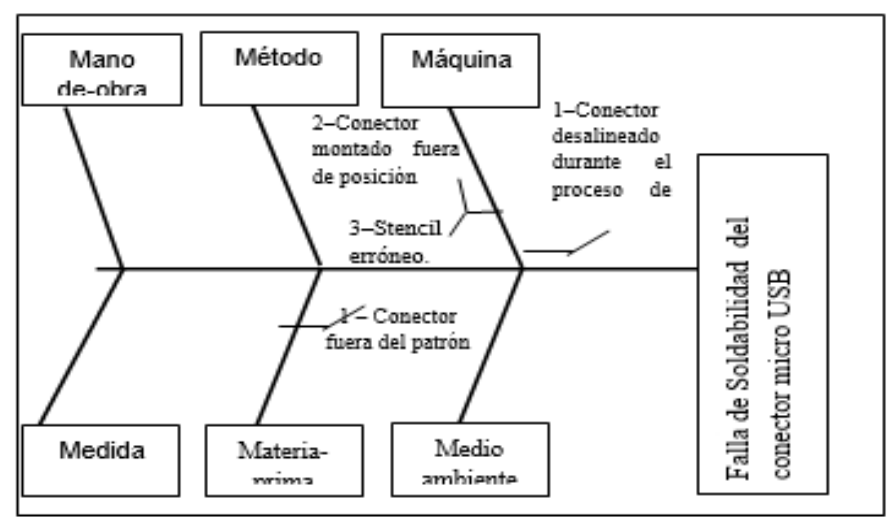

Figura 8 - Diagrama de causa y efecto - Falla de soldabilidad del conector micro USB. 
Cristiano Mourão da Fonseca, et al. / ITEGAM-JETIA Vol.02, Nº 06, pp.39-47. Junho, 2016.

-Preparación del plan de acción

En base a la identificación de la causa raíz del problema y utilizando la herramienta $5 \mathrm{~W} 2 \mathrm{H}$ fue elaborado un plan de acción conteniendo el mapeado detallado de las actividades a ser desarrolladas. El plan de acción se muestra en la tabla 2.

Tabla 2 - Diagrama 5W2H

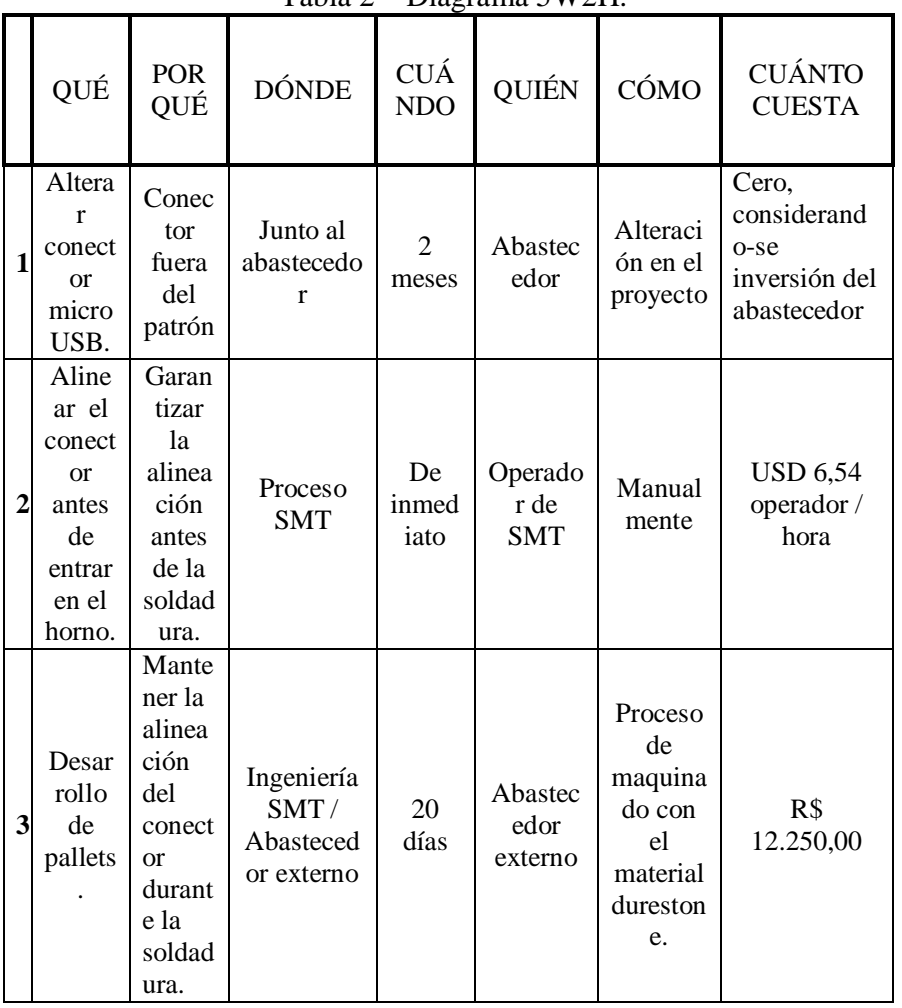

\section{III.2.2. EJECUCIÓN}

En esta etapa, fue implementado el plan de acción previamente establecido, todas las actividades fueron rigurosamente alcanzadas.

\section{$\checkmark \quad$ Desarrollo de los Pallets}

a) Material utilizado

Los pallets fueron fabricados con el material "durestone" conteniendo composites de fibra de vidrio y resina epoxi producidos a presión y alta temperatura. Fueron diseñados para atender las características de los procesos de fabricación de placas principales como:

- Baja conductividad térmica $\left(0,25 \mathrm{~W} / \mathrm{m}^{\circ} \mathrm{K}\right)$ que impide la disipación del calor para la PCB - Los componentes electrónicos poseen una temperatura máxima de contacto, en el caso de que esa temperatura sea sobrepasada los componentes pueden tener comprometida su funcionalidad.

- Material con propiedades Anti-estáticas - Muchos componentes electrónicos pueden ser dañados por descargas electrostáticas. Los materiales con propiedades electrostáticas son los que no son generadores de ese tipo de cargas.

- Mantener sus propiedades mecánicas a altas temperaturas (350 $\left.{ }^{\circ} \mathrm{C}\right)$.

- Buena resistencia a los productos químicos utilizados en el proceso de soldadura - Teniendo como ejemplo el "fluxo" que es un material utilizado para facilitar el proceso de soldadura.

- Material de fácil manipulación en ambiente de producción debido a la baja densidad, $1.90 \mathrm{~g} / \mathrm{cm}^{3}$.

b)

Diseños mecánicos

Para la fabricación de los pallets fue necesario hacer el diseño mecánico de la pieza en 3D como muestra la figura 9.

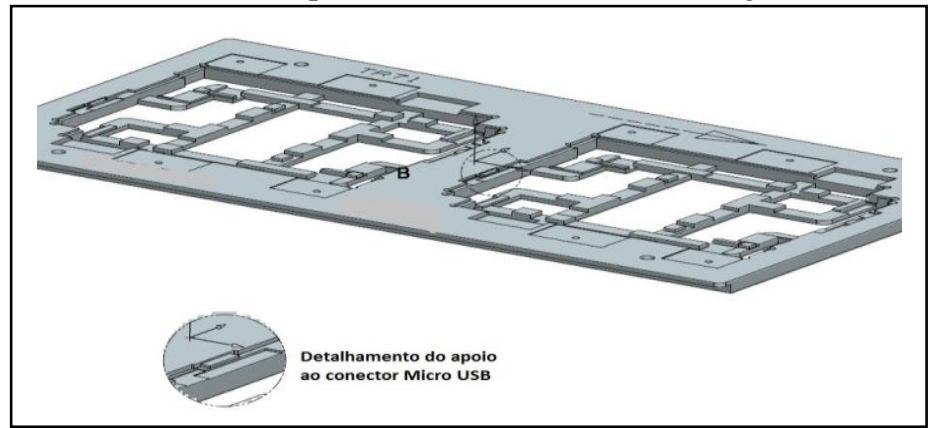

Figura 9 - Diseño mecánico del pallet en 3D.

El dimensionamiento del pallet está expuesto en la figura $10 \mathrm{y}$ fue hecho basado en los siguientes aspectos:

$\checkmark \quad$ Medidas externas: Basadas en las dimensiones de la estera interna del horno de refusión.

$\checkmark \quad$ Medidas internas: Basadas en las dimensiones de la placa principal del tablet.

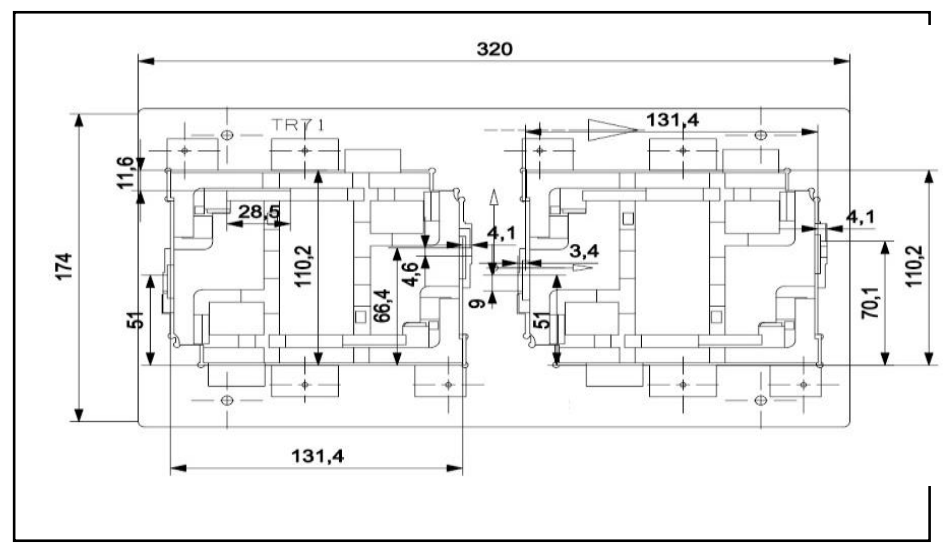

Figura 10 - Diseño mecánico del pallet en 2D con medidas en mm.

\section{III.2.3. VERIFICACIÓN}

La fase de verificación fue caracterizada por la colecta de datos en la condición final, siguiendo los mismos criterios establecidos en la fase de planeamiento como se muestra en la tabla 3. A continuación fue realizado un análisis crítico comparando los datos iniciales y finales después de la implantación del plan de acción. 
Cristiano Mourão da Fonseca, et al. / ITEGAM-JETIA Vol.02, No 06, pp.39-47. Junho, 2016.

Tabla 3 - Índice de fallas en la situación final.

\begin{tabular}{|c|c|c|}
\hline $\begin{array}{c}\text { PRODUCIÓN } \\
\text { TOTAL }\end{array}$ & $\begin{array}{c}\text { QUANTIDADE } \\
\text { DE DEFEITOS }\end{array}$ & $\begin{array}{c}\text { ÍNDICE } \\
\text { DE FALHAS }\end{array}$ \\
\hline 2400 PLACAS & 2 & $0,09 \%$ \\
\hline
\end{tabular}

\section{III.2.4 ESTANDARIZACIÓN}

En la etapa de estandarización fueron realizadas las acciones correctivas detectadas a lo largo del proceso y la unificación de todas las actividades a ser desarrolladas para que la falla no vuelva a ocurrir.

\section{ANÁLISIS, DISCUSIONES Y RESULTADOS.}

Las acciones realizadas de acuerdo a lo planeado y los resultados alcanzados para cada acción están expuestos en el cuadro 3.

Cuadro 3 - Resumen de las actividades y resultados obtenidos.

\begin{tabular}{|l|l|l|}
\hline \multicolumn{2}{|c|}{$\begin{array}{l}\text { RESUMEN DE LAS } \\
\text { ATIVIDADES }\end{array}$} & \multicolumn{1}{c|}{ RESULTADOS } \\
\hline $\mathbf{1}$ & $\begin{array}{l}\text { Fue adicionado un puesto } \\
\text { para alineación manual } \\
\text { antes del horno de } \\
\text { refusión. }\end{array}$ & $\begin{array}{l}\text { No hubo reducción significativa de los } \\
\text { indicadores generando resultados de } 12 \\
\text { \% de fallas considerados todavía } \\
\text { insatisfactorios. }\end{array}$ \\
\hline $\mathbf{2}$ & $\begin{array}{l}\text { Envío de piezas } \\
\text { defectuosas para análisis } \\
\text { del proveedor del } \\
\text { conector micro USB. }\end{array}$ & $\begin{array}{l}\text { Alteración en el proyecto del conector } \\
\text { micro USB, sin embargo con un lead } \\
\text { time de 60 días para el recibimiento de } \\
\text { las nuevas piezas. }\end{array}$ \\
\hline $\mathbf{3}$ & $\begin{array}{l}\text { Reevaluación del proceso } \\
\text { proceso, reduciendo los niveles de } \\
\text { defectos para índices evaluados como } \\
\text { aceptables de 0,09\%. }\end{array}$ \\
\hline $\mathbf{4}$ & $\begin{array}{l}\text { Evaluación del proceso } \\
\text { de fabricación de otros } \\
\text { productos. }\end{array}$ & $\begin{array}{l}\text { La utilización de los pallets fue } \\
\text { extendida a otros modelos. }\end{array}$ \\
\hline
\end{tabular}

En la actividad 1 en la cual fue adicionado un operador para realizar la alineación manual del conector antes de pasar por el horno de refusión generó un índice final de $12 \%$ de fallas, todavía muy por encima del indicador esperado de un máximo del $2 \%$. A partir de este resultado, se concluye que el la desalineación del conector micro USB está ocurriendo durante el proceso de soldadura dentro del horno de refusión.

En la actividad 2 hubo un contacto con el abastecedor de los conectores el cual solicitó el envío de piezas que presentaran la falla para un análisis más detallado. El resultado de esa investigación apunta para una falla en el proyecto del conector que estaba con dimensiones diferentes de las especificadas como muestra la figura 11 .

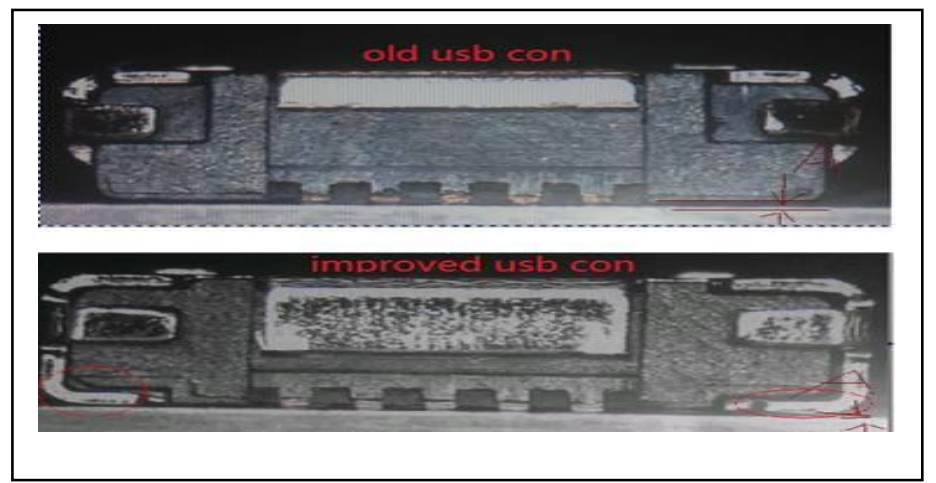

Figura 11-Conector micro USB anterior (a) y corregido (b).

Entretanto, el descubrimiento y corrección de la causa raíz del problema no atendían las necesidades de la fábrica, pues había una gran cantidad del conector considerado como defectuoso en estoque y el lead time para recibimiento del nuevo kit de material era de aproximadamente 60 días. Había también un compromiso de entrega inmediata de productos acabados a clientes súper exigentes y de esa forma se tornaba inviable aguardar el recibimiento del nuevo material del abastecedor.

Así, se hizo necesaria una acción de disposición inmediata para que la fábrica pudiera cumplir sus acuerdos comerciales. En la actividad 3 el proceso de soldadura en el horno de refusión fue reevaluado, donde se constató que los conectores micro USB no poseían el apoyo adecuado siendo movidos de su posición por la vibración de las cadenas de transporte de las placas presentes en los hornos. De esa forma, se optó entonces por desarrollar los pallets de "durestone" para garantizar la perfecta alineación de los conectores durante el proceso de soldadura. El resultado final después de la implantación de los pallets en el proceso fue la producción de 2400 placas con apenas 2 defectos y (a)

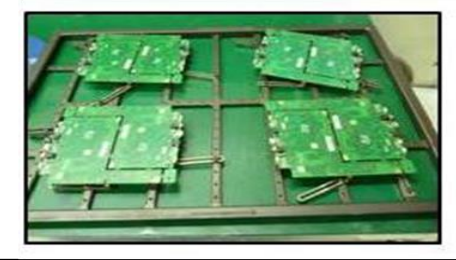

(b)

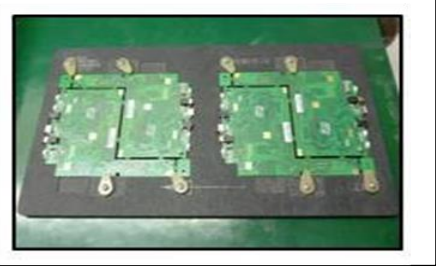

un índice de fallas de 0,09\%. La figura 12 muestra el pallet utilizado anteriormente sin el apoyo para el conector micro USB y el pallet implantado en el proceso con la corrección de la falla.

Figura 12 - (a) Pallet anterior e (b) pallet desarrollado con el apoyo al conector.

Este resultado fue considerado extremamente satisfactorio, pues el índice final quedó abajo de la meta establecida por la empresa que era del $2 \%$. Hubo una reducción del 99,3\% del número de fallas. Aún después del recibimiento de los nuevos conectores enviados por el proveedor el uso de los pallets no fue retirado del proceso, pues fue considerado un 
Cristiano Mourão da Fonseca, et al. / ITEGAM-JETIA Vol.02, № 06, pp.39-47. Junho, 2016.

dispositivo a prueba de posibles nuevas fallas de origen. Las consecuencias fueron tan positivas que la actividad 4 fue caracterizada por la extensión de la utilización del pallet en los procesos de fabricación de los otros modelos de tablets.

\section{IV.1. ANÁLISIS DE LOS COSTOS}

La cantidad de defectos generó el aumento de los costos no planificados por la empresa. Las pérdidas principales son:

$\checkmark \quad$ Mano de obra de técnicos de reparación - Cuando la falla es identificada en la etapa de pruebas funcionales, la placa es direccionada a la reparación. El costo/hora del técnico de reparación es de 10,73 USD, la media de arreglos por hora es de 5 placas y el costo de arreglo por placa es de 2,146 USD.

$\checkmark \quad$ Mano de obra de operadores de prueba - Después del arreglo técnico las placas son direccionadas nuevamente para las pruebas funcionales. El costo/hora del operador de pruebas es de 6,54 USD, la media de pruebas por hora es de 6 placas y el costo de arreglo por placa es de 1,09 USD.

$\checkmark \quad$ Compra de equipos de reparación - Para el procedimiento de reparación de estas placas fue preciso que la empresa comprara nuevos equipos como la estación de soldadura, multímetros digitales, etc. El costo total de la compra de equipos fue de 2.000,00 USD.

Conforme a lo citado anteriormente, inicialmente fueron producidas 1000 placas con un índice de fallas de 12,4\%. Después de la implantación del uso de los pallets fueron producidas 2400 placas con un índice de 0,09\%. Así, evaluándose la demanda de producción de este producto que era de 200.000 placas, calculándose el porciento de fallas de $12,4 \%$ sobre el total a ser producido se tiene que una cantidad total estimada de defectos para su arreglo será de 24.800 placas. O sea, el análisis de la falla y la implantación del uso de pallets evitaron que fuesen generadas 24.800 placas defectuosas. El total de pérdidas estimadas sobre esta cantidad de placas está listado en la tabla 4.

Tabla 4 - Valores que dejaron de ser gastados después de la implantación de los pallets.

\begin{tabular}{|l|c|c|c|}
\hline ATIVIDAD & $\begin{array}{c}\text { COSTO } \\
\text { OPERACIONAL } \\
\text { POR PLACA EN } \\
\text { USD }\end{array}$ & $\begin{array}{c}\text { TOTAL DE } \\
\text { ARACAS PARA } \\
\text { ARREGLAR }\end{array}$ & $\begin{array}{c}\text { VALOR } \\
\text { TOTAL } \\
\text { USD }\end{array}$ \\
\hline $\begin{array}{l}\text { Reparación } \\
\text { de placas }\end{array}$ & 2,146 & 24.800 & 53220,8 \\
\hline $\begin{array}{l}\text { Re-prueba } \\
\text { de placas }\end{array}$ & 1,09 & 24.800 & 27032 \\
\hline \multicolumn{3}{|c|}{ TOTAL GENERAL } & 80252,8 \\
\hline
\end{tabular}

Analizándose ahora los otros costos como la compra de equipamientos y la compra de los pallets se observa en la tabla 5 que la empresa dejó de gastar la cantidad de 70.002,80 USD.
Tabla 5 - Valores totales que dejaron de ser gastados después de la implantación de los pallets.

\begin{tabular}{|c|c|}
\hline COSTOS TOTALES & VALOR USD \\
\hline $\begin{array}{c}\text { Costos totales con reparación en } \\
24800 \text { placas (valor ahorrado) }\end{array}$ & $80.252,80$ \\
\hline $\begin{array}{c}\text { Compra de equipamientos (valor } \\
\text { ahorrado) }\end{array}$ & $2.000,00$ \\
\hline $\begin{array}{c}\text { Compra de pallets (inversión) } \\
\text { TOTAL GENERAL AHORRADO }\end{array}$ & $12.250,00$ \\
\hline
\end{tabular}

\section{CONCLUSIONES}

En este estudio fueron propuestas soluciones para el desalineamiento del conector micro USB, tales como una alteración en el proyecto específicamente de ese conector, la que sería la mejor opción. Sin embargo, esta no fue aplicada debido al largo tiempo para la conclusión de esta actividad de aproximadamente 60 días. La segunda solución y que llegó hasta ser implantada en la práctica fue la inclusión de un operador para alinear manualmente el conector micro USB antes de iniciar el proceso de soldadura en el horno de refusión. Sin embargo, los resultados obtenidos indican índices de fallas semejantes a los índices originales.

Finalmente, fue implantada la solución de utilización de pallets de durestone en el proceso de soldadura del conector micro USB en la placa principal en tablets para asegurar el perfecto alineamiento de este conector. Los objetivos del trabajo fueron alcanzados, pues hubo una reducción del índice de defectos que eran del 12,4\%, muy encima de los índices aceptables por la empresa, para los indicadores finales de 0,09\%. Haciendo una proyección sobre el total a ser producido de esta placa se verifica que la empresa dejó de generar 24.800 placas con la falla estudiada. En términos de costos y analizándose los resultados alcanzados en la tabla 5 la cual presenta que el costo operacional de reparación y re-prueba que dejó de ser desperdiciado fue de 80.252,80 USD. Evaluándose los valores totales como se describe en la tabla 8, adicionando los costos con la compra de equipamientos y reduciendo el costo de la compra de los pallets se concluye que la empresa evitó gastar 70.0002,80 USD.

De esta forma, el análisis y mejora aplicada se reflejan en factores tales como reducción de costos, aumento de la calidad, productividad y satisfacción de los clientes. La solución presentada fue extendida para otros modelos de placas. 
Cristiano Mourão da Fonseca, et al. / ITEGAM-JETIA Vol.02, № 06, pp.39-47. Junho, 2016.

\section{AGRADECIMIENTOS}

Al Instituto de Tecnología José Rocha Sérgio Cardoso (ITJC), a la Universidad Federal do Pará (UFPA) y al Instituto de Tecnología Galileo de la Amazonia (ITEGAM) por el apoyo en la realización de la investigación.

\section{REFERENCIAS BIBLIOGRÁFICAS}

[1] Paludo, Augustinho Vicente. Administração Pública: Questões, Fourth Edition, Elsevier, 2013.

[2] Montgomery, Douglas C. Introduction to Statistical Quality Control, Sixth Edition, John Wiley \& Sons, 2009.

[3] Silveira, Nilton; Pereira, Felipe; Giorgetti, Tiago. Tecnologias em sala de aula: explorando as possibilidades do tablet na educação, Anais do XI Encontro Nacional de Educação Matermática, ISSN2178-034X, 2013.

[4] Coombs, Clyde Jr. Printed Circuits Handbook. Sisxth Edition, McGrawHill, 2008.

[5] Torabi, S., Hamedi, H., Ashayeri, J. A new optimization approach for nozzle selection and component allocation in multi-heam-type SMD placement machines. Journal of Manufacturing Systems, 32 (2013) 700-714, 2013.

[6] Lee, Ning-Cheng. Reflow Soldering Processes and Troubleshootin: SMT, BGA, CSP and Flip Chip Technologies, First Edition, Newnes, 2002.

[7] Ming-Hung, Shu., Bi-Ming, Hsu., Min-Chuan, Hu. Optimal combination of soldering conditions of BGA for halogen-free and lead-free SMT-green processes, Microelectronics Reliability 52 (2012) 2690-2700, 2012.

$[8,9,10]$ Marinovic, Marcos Doro. Sistemática para implantação da garantía de qualidade em empresas montadoras de placa de circuito impresso. M.S Dissertação, Programa de Pós-Graduação em Metrologia Científica e Industrial, Universidade Federal de Santa Catarina, Santa Catarina, Brasil, 2004.

[11] Ning, JingFeng; Chen, Zhiyu; Liu, Gang. PDCA Process Application in the Continuous Improvement of Software Quality, International Conference on Computer, Mechatronics, Control and Electronic Engineering(CMCE), 10.1109/CMCE.2010.5609635, 2010.

[12] Chaves, Ana Paula; LEAL, Gislaine; HUZITA, Elisa. An Experimental Study of the FIB Framework Driven by the PDCA Cycle, International Conference of the Chilean Computer Science Society, 10.1109/SCCC.2008.10, 2008.

[13 ] Moreno, Gustavo Alberto; Jiménez Jovani Alberto. Cycle of PDCA T-Learing Model and Its Application on Interactive Digital TV, Open Journal Systems DYNA 79, no 173, 2012.
[14] Yang, Ying; Yu, Bengong; Ma, Xijun. The Process Modeling of Creating Work Breakdown Structures of Automobile R\&D Project Based on PDCA, IEEE International Conference on Service Operations and Logistics, and Informatics IEEE/SOLI, 10.1109/SOLI.2008.4686414, 2008.

[15] Juran, Joseph M.; Godfrey, A. Blanton. Juran's Quality Handbook, Fifth Edition, McGraw-Hill, 1998.

[16,17] Zhichun, Qiao; Yuejun, Xiao. Quality Improvement of Wall Energy Conservation Project Based on PDCA Cycle, International Conference on Electric Technology and Engineering(ICTCE), 10.1109/ICETCE.2011.5775312, 2011.

[18] Wong, Kam Cheong. Using an Ishikawa diagram as a tool to assist memory and retrieval of relevant medical cases from the medical literature, Journal of Medical Case Reports, 10.1186/1752-1947-5-120, 2011.

[19] Jeang, Angus; CHUNG, Chien-Ping; CHEN, Chung-Wei; LI, Huan-Chung. Optimizing process parameters of hot-bar soldering process through quality function deployment and Taguchi method, Journal of Materials Processing Technology, 209 (2009) 2967-2977, 2009.

[21] Changqing, Gao; Kezheng, Huang; Fei, Ma. Comparison of innovation methodologies and TRIZ. The Triz Journal, 2005.

[22] Truscott, Willian. Six Sigma Continual Improvement for Business. Elsevier, 2003. 